\section{P2.18 THE VALUE OF LIGHT MICROSCOPY TO DIAGNOSE UROGENITAL GONORRHOEA IN INDONESIAN CLINIC- BASED AND OUTREACH SEXUALLY TRANSMITTED INFECTIONS SERVICES}

${ }^{1}$ IPutu Yuda Hananta, ${ }^{2}$ Alje PVan Dam, ${ }^{2}$ Sylvia Maria Bruisten, ${ }^{2}$ Maarten Franciscus Schim Van Der Loeff, ${ }^{3}$ Hardyanto Soebono, ${ }^{2}$ Henry John Christiaan De Vries. ${ }^{1}$ Department of Dermatology, Academic Medical Centre, University of Amsterdam, Amsterdam, The Netherlands; ${ }^{2}$ Department of Infectious Diseases, Public Health Service of Amsterdam, Amsterdam, The Netherlands; ${ }^{3}$ Department of Dermatology and Venereology, Faculty of Medicine, Universitas Gadjah Mada, Yogyakarta - Indonesia

\subsection{6/sextrans-2017-053264.194}

Introduction Gonorrhoea is a common sexually transmitted disease caused by Neisseria gonorrhoeae $(\mathrm{Ng})$ infection. Light microscopy of urogenital smears is used as a simple tool to diagnose urogenital gonorrhoea in many resource-limited settings. We aimed to evaluate the accuracy of light microscopy to diagnose urogenital gonorrhoea as compared to a PCR based test.

Methods In 2014, we examined 632 male urethral and 360 endocervical smears in clinic-based and outreach settings in Jakarta, Yogyakarta and Denpasar, Indonesia. Using the detection of $\mathrm{Ng}$ DNA by a validated PCR as reference test, we evaluated the accuracy of two light microscopic criteria to diagnose urogenital gonorrhoea in genital smears: 1) the presence of intracellular Gram negative diplococci (IGND) and 2) $\geq 5$ polymorphonuclear leukocytes (PMNL)/oil-immersion field (oif) in urethral, or $>20 \mathrm{PMNL} /$ oif in endocervical smears.

Results In male urethral smears, IGND testing had a sensitivity, specificity, and kappa of respectively $59.0 \%, 89.4 \%$, and 0.49. For PMNL count these were respectively 59.0\%, 83.7\%, and 0.40 . The accuracy of IGND in the clinic-based settings (respectively $72.0 \%, 95.2 \%$, and 0.68 ) was better than in the outreach settings (respectively 51.2\%, 83.4\%, and 0.35). In endocervical smears, light microscopy performed poorly regardless of the setting or symptomatology, with kappas ranging from 0.09 to 0.24 .

Conclusion Light microscopy using IGND and PMNL criteria can be an option with moderate accuracy to diagnose urethral gonorrhoea among males in a clinic-based setting. The poor accuracy in detecting endocervical infections indicates an urgent need to implement advanced methods, such as PCR. Further investigations are needed to identify the poor diagnostic outcome in outreach services.

Support: This study was fully funded by Excellence Scholarship Program (Beasiswa Unggulan), Ministry of Research, Technology and Higher Education, Republic of Indonesia

\section{P2.19 PERIODICPRESUMPTIVE TREATMENT FOR VAGINAL INFECTIONS DOES NOT IMPACT THE INCIDENCE OF HIGH-RISK SUBTYPES OF HUMAN PAPILLOMA VIRUS: A SECONDARY ANALYSIS FROM THE PREVENTING VAGINAL INFECTIONS TRIAL}

${ }^{1}$ Jennifer E Balkus, ${ }^{2}$ Joshua Kimani, ${ }^{2}$ Omu Anzala, ${ }^{2}$ Emmanuel Kabare, ${ }^{2}$ Juma Shafi, ${ }^{3} \mathrm{R}$ Scott Mcclelland. ${ }^{1}$ Fred Hutchinson Cancer Research Centre, Seattle, USA; ${ }^{2}$ University of Nairobi, Nairobi, Kenya; ' University of Washington, Seattle, USA

10.1136/sextrans-2017-053264.195

Introduction Evidence suggests that women with bacterial vaginosis (BV) are at increased risk for infection with high risk sub-types of human papilloma virus (hrHPV). Among women participating in a randomised trial of periodic presumptive treatment (PPT) to reduce vaginal infections including BV, we previously reported reductions in bacterial STIs among women receiving PPT compared to placebo. In the current analysis, we assessed the effect of PPT on acquisition of HR-HPV among Kenyan women enrolled in the trial.

Methods Nonpregnant, HIV-uninfected women aged 18-45 from Kenya and the United States were randomised to receive intravaginal metronidazole $750 \mathrm{mg}$ plus miconazole $200 \mathrm{mg}$ or matching placebo for 5 consecutive nights each month for 12 months. Genital specimens were collected every other month. Following completion of the trial, enrollment and follow-up specimens from participants at the three sites in Kenya were tested for hrHPV (types 16, 18, 31, 33, 35, 39, 45, 51, 52, 56, 58, 59, 66, and 68) using the Hologic APTIMA HPV assay. Poisson regression models were used to assess the intervention effect on hrHPV incidence and time to first detection of hrHPV.

Results Among 176 Kenyan women participating in the trial, 168/176 (95\%) had specimens available for analysis (intervention $n=84$; placebo $n=84$ ). Baseline hrHPV prevalence was $17 \%(n=29)$ and similar by arm. Among participants without detectable hrHPV at enrollment, 26 participants had hrHPV detected during 114.6 person-years (incidence $=22.7 / 100$ person-years), with similar incidence in the intervention versus placebo arm (21.7/100 person-years versus 23.6/100 personyears; incidence rate ratio $[$ IRR] $=0.92,95 \%$ CI $0.42,1.99$ ).

Conclusions This intervention, which significantly reduced BV over 12 months, did not impact acquisition of hrHPV. However, the high incidence of hrHPV provides evidence in support HPV vaccination efforts in this region.

\section{P2.20 MALE PARTNER LINKAGE TO CLINIC-BASED STI/HIV SERVICES FOLLOWING A HOME-BASED COUPLE ANTENATAL EDUCATION AND TESTING INTERVENTION IN WESTERN KENYA: A RANDOMISED CONTROLLED TRIAL}

${ }^{1}$ Jennifer Mark, ${ }^{2}$ Kinuthia J, ${ }^{3}$ Osoti $A,{ }^{1}$ Krakowiak D, ${ }^{1}$ Roxby A, ${ }^{2}$ Gone Ma, ${ }^{2}$ Asila V, ${ }^{1}$ Parikh S, ${ }^{1}$ Sharma M, ${ }^{4}$ Richardson Ba, ${ }^{1}$ Farquhar C. ${ }^{1}$ University of Washington, Seattle, USA; ${ }^{2}$ Kenyatta National Hospital, Nairobi, Kenya; ${ }^{3}$ University of Nairobi, Nairobi, Kenya; ${ }^{4}$ Fred Hutchinson Cancer Research Centre, Seattle, USA

\subsection{6/sextrans-2017-053264.196}

Introduction Offering couples education and HIV rapid testing during pregnancy can increase testing of male partners, identify sero-discordant couples, and help link men to HIV care services. We aim to understand how a home-based antenatal couple HIV testing intervention affects male partner follow-up to clinic-based STI services during pregnancy.

Methods We conducted a randomised controlled trial of unaccompanied pregnant women attending a first visit at Kisumu County Hospital from September 2013 to June 2014. Women and their partners received either home-based couple education with HIV and syphilis testing during pregnancy or an invitation letter to clinic-based couple HIV testing. Men's selfreported health seeking outcomes during pregnancy were compared at 6 months postpartum.

Results Among 601 enrolled women, we reached 247 and 240 men in the intervention and control arms, respectively $(85 \%$ participation). Men who received the intervention were more likely to seek an STI consultation based on symptoms or a 
positive syphilis test $[\mathrm{RR}=1.59 ; 95 \% \mathrm{CI}=1.33-1.89]$. Postpartum, $39(16 \%)$ intervention and 21 (9\%) control men reported known HIV-positive status during pregnancy. Despite increased knowledge of HIV-positive status within the intervention group, men were less likely to link to HIV care services $[\mathrm{RR}=0.69 ; 95 \% \mathrm{CI}=0.50-0.96]$, as $41 \%$ (16 of 39$)$ of men were newly diagnosed with HIV. No differences were observed for uptake of male circumcision within the study period $[\mathrm{RR}=1.59 ; 95 \% \mathrm{CI}=0.89-2.87]$ or attendance of subsequent clinic-based antenatal care with the female partner $[\mathrm{RR}=1.11 ; 95 \% \mathrm{CI}=0.85-1.41]$.

Conclusion Home-based couple education and testing resulted in greater uptake of clinic-based STI consultation services among men. However, increased knowledge of HIV-status in the home did not lead to increased HIV care service uptake for men, potentially because there was a greater proportion of men with new HIV diagnoses in the intervention arm. This group of newly diagnosed men should be targeted with research to increase linkage and engagement to HIV care.

\section{P2.21 FEASIBILITY STUDY FOR THE DEVELOPMENT OF A RAPID LATERAL FLOW POINT OF CARE TEST FOR CONGENITAL SYPHILIS}

${ }^{1} J o h n$ Deutsch, 'Yetunda F Fakile, ${ }^{1}$ Ellen Kersh, ${ }^{2} J a v a n$ Esfandiari, ${ }^{3}$ Ardianne Cruz. ${ }^{1}$ The Centre for Disease Control and Prevention, Atlanta, GA, USA; ${ }^{2}$ Chembio Diagnostic Systems, Medford, NY, USA; ${ }^{3}$ UCONN Health Centre, Farmington, CT, USA

10.1136/sextrans-2017-053264.197

Introduction The World Health Organisation (WHO) estimates there are 713600 to $1,575,000$ cases of congenital syphilis worldwide annually. Congenital syphilis can lead to several health conditions at birth. Currently there is no rapid point of care test available for its diagnosis.Here we introduce a novel rapid point of care test for congenital syphilis. The objective of this test includes the ability to test whole blood or serum taken from the infant or cord blood while blocking the mother's IgG antibodies.

Methods Thirty-four (34) syphilis positive and negative gestational serum samples and five (5) positive congenital syphilis samples, were obtained from University of Connecticut Health Centre. Twenty-two (22) samples were RPR reactive. Chembio DPP Syphilis IgG and IgM test devices were obtained from the manufacturer. The required number of $\operatorname{IgG}$ and IgM devices were modified at the CDC to include an IgG blocker in the device to produce four different assay devices used for this study. Each serum sample was tested in the four separate devices. Results were reported based on the patterns observed in the assay windows.

Results All (17/17) the RPR positive gestational samples were positive (100\%), and 5 of 5 congenital positive samples were positive (100\%) using the IgM device with IgG blocking agent. Only $5 / 17$ of gestational samples $(29.4 \%)$ and $1 / 5$ congenital samples (20\%) were positive using the IgM device without $\operatorname{IgG}$ blocking agent. For the $\operatorname{IgG}$ device with the $\operatorname{IgG}$ blocking agent all samples were negative. The IgG device without the blocking reagent resulted in the detection of 19/ $22(86.4 \%)$ of the RPR positive samples. With the exception of one sample all negative samples were negative by all 4 test devices.

Conclusion Only a limited number of positive and negative gestational and congenital syphilis samples were evaluated in this study, due to the short supply of such rare samples. It is apparent from this limited data that by blocking the mother's IgG an increase in sensitivity can be achieved. More in depth studies are necessary in order to prove the concept.

\section{P2.22 SURVEILLANCE OF HIV-1 TRANSMITTED DRUG RESISTANCE AMONG DRUG-NAÏVE POPULATIONS IN RIO DE JANEIRO, BRAZIL}

${ }^{1}$ José Carlos Couto Fernandez, ${ }^{2}$ Giovanni Ravasi, ${ }^{3}$ Michelle Das Neves, ${ }^{3}$ Jose Henrique Pilotto, ${ }^{3}$ Carlos Silva de Jesus, ${ }^{3}$ Mariza Gonçalves Morgado. 'Laboratory of AIDS and Molecular Immunology, Oswaldo Cruz Foundation-loc/Fiocruz, Rio de Janeiro - RJ, Brazil; ${ }^{2}$ Pan-American Health Organization-PAHO, World Health Organisation, Washington DC, USA; ${ }^{3}$ Oswaldo Cruz Foundation-loc/Fiocruz, Rio de Janeiro - RJ, Brazil

\subsection{6/sextrans-2017-053264.198}

Introduction The WHO Global HIV drug resistance network (HIV-ResNet), was stablished to monitor the emergence and help to control the transmission of HIV-1 drug resistant strains. The TDR is progressively increasing over the last years in some Brazilian regions, mainly where the epidemic is concentrated. This study evaluated the trends in the prevalence of TDR mutations and dynamic of subtypes, among drug-naïve HIV-1 infected individuals from vulnerable group populations in the context of the WHO HIV-ResNet.

Methods We analysed a total of 536 HIV-1 sequences collected during 2005 to 2014, targeting drug naïve pregnant woman from four public antenatal care units and 159 recently diagnosed $(<1$ year) individuals identified in VCTs in all Rio de Janeiro state. The profiles of TDR mutations were evaluated using the updated WHO transmitted resistance mutation list and HIV-1 genetic diversity evaluated by phylogenetic analysis.

Results Overall, the prevalence of TDR was 12.5\% (CI95\%, $7.15 \%$ to $16.5 \%)$ being, $5.8 \%(\mathrm{CI} 95 \%, 2.5 \%$ to $9.15 \%)$ to the nucleoside reverse transcriptase inhibitors (NRTIs), 3\% (CI95\%, $0.1 \%$ to $4.85 \%)$ to non-nucleoside inhibitors (NNRTIs) and $3.7 \%$ (CI95\%, $1.24 \%$ to $7.5 \%)$ to protease inhibitors (PIs). Both studied groups showed similar TDR prevalence for all drug classes. The timidine-associated mutations (TAMs) and M184V were the most prevalent TDR mutations found in RT gene, followed by K103N, T215 revertants and F77L. The M46I PI associated mutation was the more frequent, followed by V82A and L90M. HIV-1 subtype B was the most prevalent (80\%), followed by F1 (7.5\%), subtype $\mathrm{C}(4 \%)$ and $\mathrm{BF}$ recombinants (3.5\%). In addition to non-B HIV-1 subtype A1, G, CRF02_AG, CRF31_BC, FC and DF recombinants, identified in $4 \%$ of genotyped samples. Significant difference was observed in the two groups, where subtype $F(12 \%)$ was more prevalent in pregnant woman, while subtype $\mathrm{C}$ prevalent in new diagnosed subjects (5.9\%). Conclusion This work tried to study trends of HIV-1 TDR and the genetic diversity in Rio de Janeiro state, the second major HIV/AIDS epidemic in Brazil. The results demonstrated 